\title{
Calcaneal Apophysitis (Sever's Disease): MRI Findings
}

\author{
Dogan $\mathrm{MS}^{1}$, Doganay $\mathrm{S}^{2}$, Koc $\mathrm{G}^{3}$, Gorkem $\mathrm{SB}^{4}$, Ciraci $\mathrm{S}^{5}$, Coskun $\mathrm{A}^{6}$
}

\begin{abstract}
Calcaneal apophysitis (Sever's disease), is the most frequent cause of the heel pain in growing children. The diagnosisis generally made with a typical history and clinical examination. Imaging modalities including radiography and magnetic resonance imaging can help establish the diagnosis. In this case we report imaging findings of an 11-year-old boy diagnosed as Sever's disease.
\end{abstract}

\section{Introduction}

Calcaneal apophysitis (Sever's disease), is the most frequent cause of the heel pain in physically active growing children ${ }^{1,2,3,4,5}$. The diagnosis of Sever's disease is generally made with the typical history of posterior heel pain that exacerbates with physical activity and clinical examination findings of no or mild swelling without any local skin manifestations like eryhtema or edema ${ }^{1,2,3}$. Although the requirement of using radiological methods for the diagnosis of Sever's disease is controversial, foot radiographs are used to be obtained as a part of the routine clinical evaluation ${ }^{1,4,5}$. Moreover, imaging methods could be useful in order to exclude other pathologies like fracture, tumors, and osteomyelitis ${ }^{6}$. However, radiography findings of this disease may be also seen as a variant of normal ${ }^{3,4}$. Therefore, magnetic resonance imaging (MRI) can play a crucial role in the diagnosis of relevant cases ${ }^{6,7}$. We herein report an 11 -year-old boy diagnosed as Sever's disease with both radigraphy and MRI findings.

\section{The Case}

An 11-year-old boy was admitted to our hospital with the complaint of the right heel pain that exacerbates with the physical activity for the last two weeks. The pain initiated spontaneously without any history of trauma. There were no associated complaints or history of chronic illness. Physical examination was normal except tenderness of right heel. Laboratory tests were within normal limits as following; haemoglobin $13.8 \mathrm{~g} / \mathrm{dl}$, white cell count $5 \times 10^{3} / \mu \mathrm{l}$, platelet $300 \times 10^{3} / \mu$ l, erythrocyte sedimentation rate $16 \mathrm{~mm} / \mathrm{hr}, \mathrm{C}$ reactive protein $3.98 \mathrm{mg} / \mathrm{dl}$. In the light of the clinical and laboratory findings, Sever's disease was considered as a preliminary diagnosis due to presence of the isolated tenderness and heel pain on physical examination without any history of trauma and chronic illness, and accompanying unremarkable laboratory findings. Radiographs of
'Mehmet S Dogan, Paediatric Radiologist, MD Radiology Clinic, Edirne Sultan 1, Murat State Hospital, Turkey, ${ }^{2}$ Selim Doganay, MD, Assoc. Prof., Department of Radiology, Erciyes University, Faculty of Medicine, Turkey, ${ }^{3}$ Gonca Koc, MD, Paediatric Radiologist, Department of Radiology, Erciyes University, Faculty of Medicine, Turkey, ${ }^{4}$ Sureyya B Gorkem, MD, Assist Prof., Department of Radiology, Erciyes University, Faculty of Medicine, Turkey, ${ }^{5}$ Saliha Ciraci MD, Paediatric Radiology Fellow, Department of Radiology, Erciyes University, Faculty of Medicine, Turkey, ${ }^{6}$ Abdulhakim Coskun, MD, Prof. Department of Radiology, Erciyes University, Faculty of Medicine, Turkey.

Address for correspondence:

Mehmet S Dogan

E-mail: msaitdogan@hotmail.com

\section{How to cite}

Dogan MS, Doganay S, Koc G, Gorkem SB, Ciraci S, Coskun A. Calcaneal Apophysitis (Sever's Disease): MRI Findings. J Nepal Paediatr Soc 2015;35(2):172174.

doi: http://dx.doi.org/10.3126/jnps.v35i2.13873

This work is licensed under a Creative Commons Attribution 3.0 License.

\section{(c) (i)}

the bilateral feet and magnetic resonance imaging (MRI) of the right foot were obtained to exclude other pathologies causing heel pain. Radiographs revealed increased density and fragmentation of calcaneal apophysis on both sides (Figure 1). 


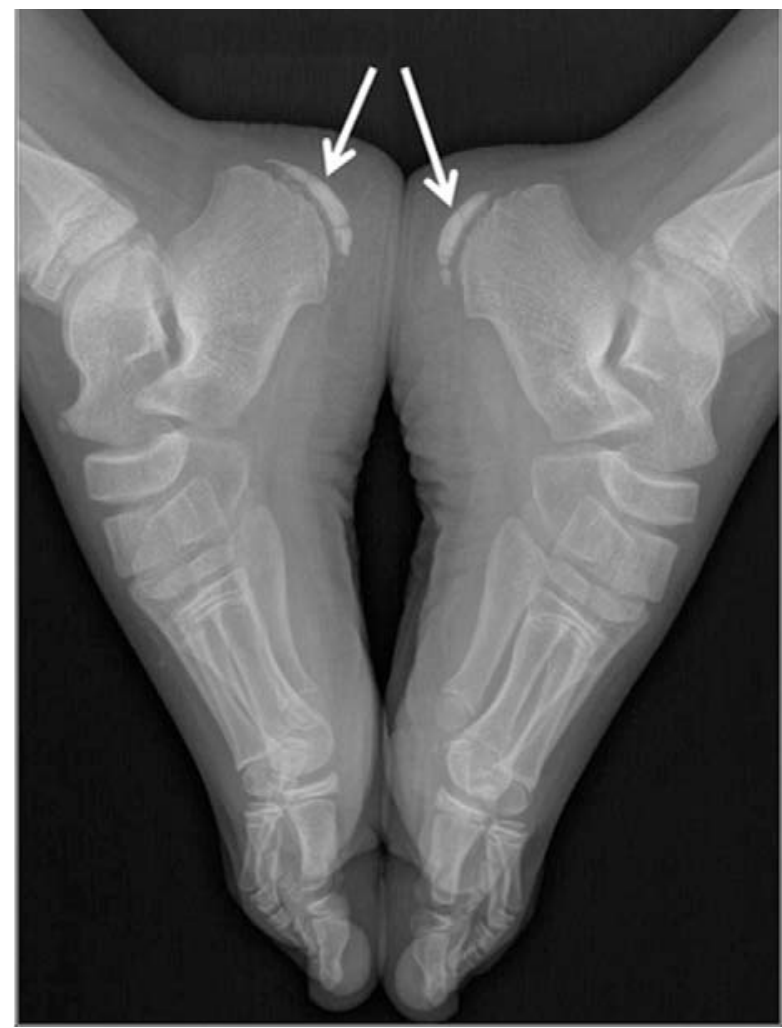

Fig 1: Radiographs revealed increased density and fragmentation of calcaneal apophysis on both sides (arrows).
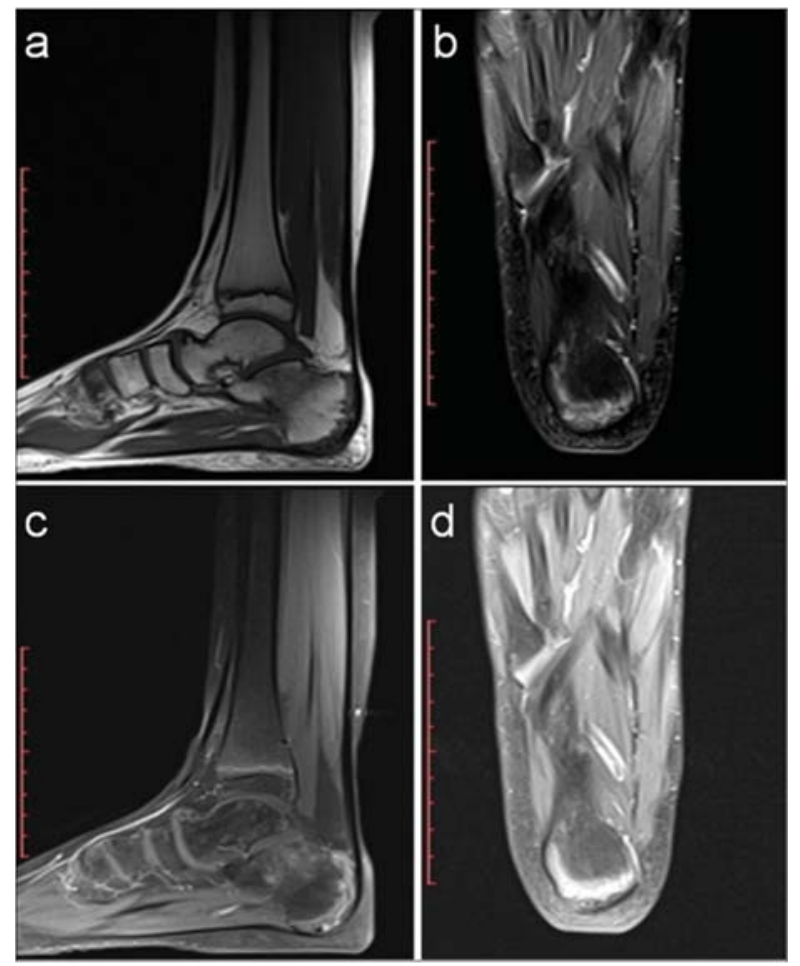

Fig 2: Fragmentation of calcaneal apophysis was seen on sagittal T1 weighted image (a), hyperintensity consistent with bone marrow edema on axial fat saturated T2 image (b), enhancement after contrast administration on sagittal (c) and axial (d) fat saturated T1 images were also shown.
MRI showed findings of the right calcaneal apophysitis including; fragmentation, which was better seen on T1 weighted images, hyperintensity consistent with oedema on fat saturated T2 weighted images and enhancement following contrast administration on fat saturated T1 images (Figure 2).

The radiographs and $\mathrm{MRI}$ were otherwise unremarkable. Therefore, the diagnosis of Sever's disease was established. He was recommended to restrict physical activities and prescribed oral antiinflammatory drugs.

\section{Discussion}

Sever's disease is thought to occur due to repetitive microtrauma caused by traction on the adhesion site of the Achilles tendon to the calcaneal tuberosity ${ }^{4,7}$. This is the most frequent cause of the heel pain in children and the diagnosis is usually made with clinical history and physical examination ${ }^{1,2,3,4,5}$. Patients often suffer from chronic heel pain and approximately in $60 \%$ of the cases complaints are associated with both heels. The average age at presentation is 11 years, ranging from 8 to 15 years. It is more commonly seen in boys with a 2-3:1, male:female ratio ${ }^{2,4}$. Differential diagnosis list of the heel pain in a child may also include other conditions like fracture, osteomyelitis, Achilles tendonitis, retrocalcaneal bursitis, calcaneal cysts, tumors, and plantar fasciitis ${ }^{5,7}$. During the clinical evaluation, foot radiographs are generally obtained for excluding other pathologies ${ }^{6}$. Although fragmentation and increased density of the calcaneal apophysis observed in radiographs are defined as radiographic findings of Sever's disease, these findings are not pathognomonic for the diagnosis and can be also seen in healthy children ${ }^{1,2,4,5}$. MRI is another useful imaging modality which can be implemented in exclusion of other causes of heel pain particularly in doubtful cases $5,6,7$. Inflammatory findings like bone marrow edema and enhancement after gadolinium administration within the calcaneal apophysis can be detected with $\mathrm{MRI}^{6}$. Anti-inflammatory medications and restriction of physical activity are the treatment of choice in such cases ${ }^{4}$.

\section{Conclusion}

In conclusion, Sever's disease should be kept in mind in physically active growing children when the heel pain is present. In relevant cases implementation of MRI may help to establish the diagnosis. 


\section{References}

1. Rachel JN, Williams JB, Sawyer JR, Warner WC, Kelly DM. Is radiographic evaluation necessary in children with a clinical diagnosis of calcaneal apophysitis (sever disease)? J Pediatr Orthop 2011;31(5):548-50.

2. Sitati FC, Kingori J. Chronic bilateral heel pain in a child with Sever disease: case report and review of literature. Cases J 2009;21;2:9365.

3. Acu B, Kara T, Asci ST, Altunkas A. MRI findings of a case with bilateral calcaneal apophysitis. J Contemp Med. 2014; 4 (Supplement Case Reports) CR-29-CR-31.
4. Hussain S, Hussain K, Hussain S, Hussain S. Sever's disease: a common cause of paediatric heel pain. BMJ Case Rep 2013;27;2013

5. Kose O, Celiktas M, Yigit S, Kisin B. Can we make a diagnosis with radiographic examinationalone in calcaneal apophysitis. J Pediatr Orthop B 2010;19(5):396-98.

6. Lawrance DA, Rolen MF, Morshed KA, Moukaddam H. MRI of Heel Pain. AJR 2013;200:845-55.

7. Mustapić M, Borić I, Lepur D, Zadravec D, Visković K. Sever's disease complicated with osteomyelitis. Acta Clin Croat 2014;53(2):252-55. 